\title{
Positive Solutions of a Singular System with Two Point Coupled Boundary Conditions
}

\author{
Naseer Ahmad Asif \\ Department of Mathematics, School of Science and Technology, University of Management and Technology, Lahore, Pakistan
}

Email address:

naseerasif@yahoo.com (N. A. Asif)

To cite this article:

Naseer Ahmad Asif. Positive Solutions of a Singular System with Two Point Coupled Boundary Conditions. American Journal of Applied Mathematics. Special Issue: Proceedings of the 1st UMT National Conference on Pure and Applied Mathematics (1st UNCPAM 2015). Vol. 3, No. 3-1, 2015, pp. 19-24. doi: 10.11648/j.ajam.s.2015030301.14

\begin{abstract}
In this paper, we study the existence of positive solutions to a system of nonlinear differential equations subject to two-point coupled boundary conditions. Further, the nonlinearities are allowed to be singular with respect to first order derivatives. An example is included to show the applicability of our result.
\end{abstract}

Keywords: Positive Solutions, Coupled System, Singular Ordinary Differential Equations, Coupled Boundary Conditions

\section{Introduction}

Coupled boundary conditions arises in the study of reaction-diffusion equations and Sturm-Liouvillie problems, see $[3,4,11]$ and Chapter 13 of [19]. The study of elliptic systems with coupled boundary conditions was initiated by Agmon and coauthors [2]. In [2], the authors studied elliptic systems with the following type of coupled boundary conditions

$$
\left.A f\right|_{\partial \Omega}+B \frac{\partial f}{\partial v}
$$

where $A$ and $B$ are differential operators from $L^{2}(\Omega ; W)$ to $L^{2}(\partial \Omega ; W), \Omega \subset \mathbb{R}^{n}$ and $W$ is a separable Hilbert space. Mehmeti [14], Mehmeti, Nicaise [15] and Nicaise [16] have been studied coupled boundary conditions in the study of interaction problems and elliptic operators on polygonal domains. Coupled boundary conditions have also some applications in Mathematical Biology. For example, Leung [11] studied the following reaction-diffusion system for preypredator interaction:

$$
\begin{gathered}
\frac{\partial u_{1}}{\partial t}(t, x)=\sigma_{1} \Delta u_{1}+u_{1}\left(a+f_{1}\left(u_{1}, u_{2}\right)\right), \\
\frac{\partial u_{2}}{\partial t}(t, x)=\sigma_{2} \Delta u_{2}+u_{2}\left(-r+f_{2}\left(u_{1}, u_{2}\right)\right), \\
t \geq 0, x \in \Omega \subset \mathbb{R}^{n},
\end{gathered}
$$

subject to the coupled boundary conditions

$$
\frac{\partial u_{1}}{\partial \eta}=0, \frac{\partial u_{2}}{\partial \eta}-p\left(u_{1}\right)-q\left(u_{2}\right)=0 \text { on } \partial \Omega
$$

where $\Delta=\sum_{i=1}^{n} \frac{\partial^{2}}{\partial x_{i}^{2}}, a, r, \sigma_{1}, \sigma_{2}$ are positive constants, $f_{1}, f_{2}: \mathbb{R}^{2} \rightarrow \mathbb{R}$ have Holder continuous partial derivatives up to second order in compact sets, $\eta$ is a unit outward normal at $\partial \Omega$ and $p$ and $q$ have Holder continuous first derivatives in compact subsets of $[0, \infty)$. The functions $u_{1}(t, x), u_{2}(t, x)$ respectively represent the density of prey and predator at time $t \geq 0$ and at position $x=\left(x_{1}, \ldots, x_{n}\right)$. Similar coupled boundary conditions are also studied in [5] for biochemical system. All the above mention works are related to coupled boundary conditions for partial differential equations. To the best of our knowledge, we believe that coupled system of nonlinear ordinary differential equations subject to coupled boundary conditions have never been studied previously.

For the study of existence theory for boundary value problems, we refer the readers to the recent papers $[6,7,9$, $10,12,13,17,18]$. An excellent resource on singular boundary value problems with an extensive bibliography was produced by Agarwal and O'Regan [1].

Recently, the author and coauthors [8] have studied the existence of positive solution to the following system under certain assumptions 


$$
\begin{aligned}
& -x^{\prime \prime}(t)=p(t) f\left(t, y(t), x^{\prime}(t)\right), \quad t \in(0,1), \\
& -y^{\prime \prime}(t)=q(t) g\left(t, x(t), y^{\prime}(t)\right), \quad t \in(0,1), \\
& a_{1} x(0)-b_{1} x^{\prime}(0)=0, x^{\prime}(1)=0, \\
& a_{2} y(0)-b_{2} y^{\prime}(0)=0, y^{\prime}(1)=0,
\end{aligned}
$$

The present paper is a generalization of [8] to a more general coupled singular system subject to coupled boundary conditions. We study existence of positive solutions to the following system of boundary value problems

$$
\begin{aligned}
& -x^{\prime \prime}(t)=p(t) f\left(t, x(t), y(t), x^{\prime}(t)\right), \quad t \in(0,1), \\
& -y^{\prime \prime}(t)=q(t) g\left(t, x(t), y(t), y^{\prime}(t)\right), \quad t \in(0,1), \\
& a_{1} x(0)-b_{1} y^{\prime}(0)=0, x^{\prime}(1)=0, \\
& a_{2} y(0)-b_{2} x^{\prime}(0)=0, y^{\prime}(1)=0,
\end{aligned}
$$

where the

nonlinearities $f, g:[0,1] \times[0, \infty) \times[0, \infty) \times(0, \infty) \rightarrow[0, \infty)$ are continuous and are allowed to be singular at $x^{\prime}=0, y^{\prime}=0$; $p, q \in C(0,1), p>0$ and $q>0$ on $(0,1)$. By singularity we mean that the functions $f(t, x, y, z), g(t, x, y, z)$ are allowed to be unbounded at $z=0$. Here, we study existence of at least one $C^{1}$-positive solution for the system (1.5) under more general and simple assumptions as compared to the assumptions in [8] for (1.4).

By a $C^{1}$-positive solution of the system (1.5), we mean that $(x, y) \in\left(C^{1}[0,1] \cap C^{2}(0,1)\right) \times\left(C^{1}[0,1] \cap C^{2}(0,1)\right),(x, y)$ satisfies (1.5), $x>0, y>0$ on $[0,1]$ and $x^{\prime}>0, y^{\prime}>0$ on $[0,1)$.

Throughout the paper, assume that the following conditions hold:

(A1) $p, q \in C(0,1), p, q>0$ on $(0,1), \quad \int_{0}^{1} p(t) d t<+\infty$ and $\int_{0}^{1} q(t) d t<+\infty$

(A2) $f, g:[0,1] \times[0, \infty) \times[0, \infty) \times(0, \infty) \rightarrow[0, \infty) \quad$ are continuous with $f(t, x, y, z)>0$ and $g(t, x, y, z)>0$ on $[0,1] \times(0, \infty) \times(0, \infty) \times(0, \infty)$;

$$
f(t, x, y, z) \leq h_{1}(x) k_{1}(y)\left(u_{1}(z)+v_{1}(z)\right)
$$

and $g(t, x, y, z) \leq h_{2}(x) k_{2}(y)\left(u_{2}(z)+v_{2}(z)\right)$, where $u_{i}(i=1,2)>0$ are continuous and nonincreasing on $(0, \infty)$, $h_{i}(i=1,2) \geq 0, k_{i}(i=1,2) \geq 0, v_{i}(i=1,2) \geq 0$ are continuous and nondecreasing on $[0, \infty)$;

$$
\begin{gathered}
\sup _{c \in(0, \infty)} \frac{c}{\left(1+\frac{b_{2}}{a_{2}}\right) I^{-1}\left(h_{1}(c) k_{1}(c) \int_{0}^{1} p(t) d t\right)}>1, \\
+\left(1+\frac{b_{1}}{a_{1}}\right) J^{-1}\left(h_{2}(c) k_{2}(c) \int_{0}^{1} q(t) d t\right)
\end{gathered}
$$

where

$$
I(\mu)=\int_{0}^{\mu} \frac{d \tau}{u_{1}(\tau)+v_{1}(\tau)}, J(\mu)=\int_{0}^{\mu} \frac{d \tau}{u_{2}(\tau)+v_{2}(\tau)}, \text { for } \mu>0
$$

(A5) $I(\infty)=\infty$ and $J(\infty)=\infty$;

(A6) for real constants $M>0$ and $L>0$ there exist continuous functions $\varphi_{M L}$ and $\psi_{M L}$ defined on [0,1] and positive on $(0,1)$, and constants $0 \leq \gamma_{1}, \delta_{1}, \gamma_{2}, \delta_{2}<1$ satisfying $\quad\left(1-\gamma_{1}\right)\left(1-\gamma_{2}\right) \neq \delta_{1} \delta_{2}, \quad$ such that $f(t, x, y, z) \geq \varphi_{M L} x^{\gamma_{1}} y^{\delta_{1}} \quad$ and $\quad g(t, x, y, z) \geq \psi_{M L} x^{\gamma_{2}} y^{\delta_{2}} \quad$ on $[0,1] \times[0, M] \times[0, M] \times[0, L]$;

$$
\int_{0}^{1} p(t) u_{1}\left(C \int_{t}^{1} p(s) \varphi_{M L}(s) d s\right) d t<+\infty
$$

and $\int_{0}^{1} q(t) u_{2}\left(C \int_{t}^{1} q(s) \psi_{M L}(s) d s\right) d t<+\infty$ for any real constant $C>0$.

Remark 1.1. Since $I, J$ are continuous, $I(0)=0$, $I(\infty)=\infty, J(0)=0, J(\infty)=\infty$, and are monotone increasing. Hence, $I$ and $J$ are invertible. Moreover, $I^{-1}$ and $J^{-1}$ are also monotone increasing.

\section{Main Result: Existence of at Least One Positive Solutions Introduction}

Theorem 2.1. Assume that (A1) - (A7) hold. Then, the system (1.5) has at least one $C^{1}$-positive solution.

Proof. In view of (A4), we can choose real constant $M>0$ such that

$$
\frac{M}{r}>1
$$

where

$$
\begin{aligned}
r & =\left(1+\frac{b_{2}}{a_{2}}\right) I^{-1}\left(h_{1}(c) k_{1}(c) \int_{0}^{1} p(t) d t\right) \\
& +\left(1+\frac{b_{1}}{a_{1}}\right) J^{-1}\left(h_{2}(c) k_{2}(c) \int_{0}^{1} q(t) d t\right) .
\end{aligned}
$$

From the continuity of $I$ and $J$, we choose $\varepsilon>0$ small enough such that

$$
\begin{aligned}
& \frac{M}{\left(1+\frac{b_{2}}{a_{2}}\right) I^{-1}\left(h_{1}(M) k_{1}(M) \int_{0}^{1} p(s) d s+I(\varepsilon)\right)}>1 . \\
& +\left(1+\frac{b_{1}}{a_{1}}\right) J^{-1}\left(h_{2}(M) k_{2}(M) \int_{0}^{1} q(s) d s+J(\varepsilon)\right)
\end{aligned}
$$

Choose a real constant $L>0$ such that

$$
L>\max \left\{\begin{array}{l}
I^{-1}\left(h_{1}(M) k_{1}(M) \int_{0}^{1} p(s) d s+I(\varepsilon)\right), \\
J^{-1}\left(h_{2}(M) k_{2}(M) \int_{0}^{1} q(s) d s+J(\varepsilon)\right)
\end{array}\right\}
$$

Choose $n_{0} \in\{1,2, \ldots\}$ such that $\frac{1}{n_{0}}<\varepsilon$. For each 
$n \in\left\{n_{0}, n_{0}+1, \ldots\right\}$, define retractions $\theta: \mathbb{R} \rightarrow[0, M]$ and $\rho: \mathbb{R} \rightarrow[1 / n, L]$ by

$$
\theta(x)=\max \{0, \min \{x, M\}\}
$$

and

$$
\rho(x)=\max \{1 / n, \min \{x, L\}\}
$$

Consider the modified system of BVPs

$-x^{\prime \prime}(t)=p(t) f\left(t, \theta(x(t)), \theta(y(t)), \rho\left(x^{\prime}(t)\right)\right), \quad t \in(0,1)$,

$-y^{\prime \prime}(t)=q(t) g\left(t, \theta(x(t)), \theta(y(t)), \rho\left(y^{\prime}(t)\right)\right), \quad t \in(0,1)$,

$a_{1} x(0)-b_{1} y^{\prime}(0)=0, x^{\prime}(1)=\frac{1}{n}$,

$$
a_{2} y(0)-b_{2} x^{\prime}(0)=0, y^{\prime}(1)=\frac{1}{n},
$$

Since $\quad f\left(t, \theta(x(t)), \theta(y(t)), \rho\left(x^{\prime}(t)\right)\right)$ $g\left(t, \theta(x(t)), \theta(y(t)), \rho\left(y^{\prime}(t)\right)\right)$ are continuous and bounded on $[0,1] \times \mathbb{R}^{3}$, by Schauder's fixed point theorem, it follows that the modified system of BVPs (2.4) has a solution $\left(x_{n}, y_{n}\right) \in\left(C^{1}[0,1] \cap C^{2}(0,1)\right) \times\left(C^{1}[0,1] \cap C^{2}(0,1)\right)$. Using (2.4) and (A2), we obtain

$$
x_{n}^{\prime \prime}(t) \leq 0 \text { and } y_{n}^{\prime \prime}(t) \leq 0 \text { for } t \in(0,1),
$$

which on integration from $t$ to 1 , use the boundary conditions (BCs), implies that

$$
x_{n}^{\prime}(t) \geq \frac{1}{n} \text { and } y_{n}^{\prime}(t) \geq \frac{1}{n} \text { for } t \in[0,1]
$$

Integrating (2.5) from 0 to $\mathrm{t}$, using the BCs and (2.5), we have

$$
x_{n}(t) \geq\left(t+\frac{b_{1}}{a_{1}}\right) \frac{1}{n} \text { and } y_{n}(t) \geq\left(t+\frac{b_{2}}{a_{2}}\right) \frac{1}{n} \text { for } t \in[0,1]
$$

From (2.5) and (2.6), it follows that

$$
\left\|x_{n}\right\|=x_{n}(1) \text { and }\left\|y_{n}\right\|=y_{n}(1), \text { where }\|u\|=\max _{t \in[0,1]}|u(t)| \text {. }
$$

Now, we show that

$$
x_{n}^{\prime}(t)<L, y_{n}^{\prime}(t)<L, t \in[0,1]
$$

First, we prove $x_{n}^{\prime}(t)<L$ for $t \in[0,1]$. Suppose $x_{n}^{\prime}\left(t_{1}\right) \geq L$ for some $t_{1} \in[0,1]$. Using (2.4) and (A3), we have

$$
\begin{aligned}
& -x_{n}^{\prime \prime}(t) \leq p(t) h_{1}\left(\theta\left(x_{n}(t)\right)\right) k_{1}\left(\theta\left(y_{n}(t)\right)\right) \\
& \left(u_{1}\left(\rho\left(x_{n}^{\prime}(t)\right)\right)+v_{1}\left(\rho\left(x_{n}^{\prime}(t)\right)\right)\right), \quad t \in(0,1),
\end{aligned}
$$

which implies that

$$
\frac{-x_{n}^{\prime \prime}(t)}{u_{1}\left(\rho\left(x_{n}^{\prime}(t)\right)\right)+v_{1}\left(\rho\left(x_{n}^{\prime}(t)\right)\right)} \leq h_{1}(M) k_{1}(M) p(t), \quad t \in(0,1) .
$$

Integrating from $t_{1}$ to 1 , using the $\mathrm{BCs}$, we obtain

$$
\int_{\frac{1}{n}}^{x_{n}^{\prime}\left(t_{1}\right)} \frac{d z}{u_{1}(z)+v_{1}(z)} \leq h_{1}(M) k_{1}(M) \int_{t_{1}}^{1} p(t) d t
$$

which can also be written as

$$
\int_{\frac{1}{n}}^{L} \frac{d z}{u_{1}(z)+v_{1}(z)}+\int_{L}^{x_{n}^{\prime}\left(t_{1}\right)} \frac{d z}{u_{1}(L)+v_{1}(L)} \leq h_{1}(M) k_{1}(M) \int_{0}^{1} p(t) d t .
$$

Using the increasing property of $I$, we obtain

$$
I(L)+\frac{x_{n}^{\prime}\left(t_{1}\right)-L}{u_{1}(L)+v_{1}(L)} \leq h_{1}(M) k_{1}(M) \int_{0}^{1} p(t) d t+I(\varepsilon),
$$

and using the increasing property of $I^{-1}$, leads to

$$
L \leq I^{-1}\left(h_{1}(M) k_{1}(M) \int_{0}^{1} p(t) d t+I(\varepsilon)\right)
$$

Which is a contradiction to (2.3). Hence, $x_{n}^{\prime}(t)<L$ for $t \in[0,1]$.

Similarly, we can show that $y_{n}^{\prime}(t)<L$ for $t \in[0,1]$.

Now, we show that

$$
\left\|x_{n}\right\|+\left\|y_{n}\right\|<M
$$

Suppose $\left\|x_{n}\right\|+\left\|y_{n}\right\| \geq M$. From (2.4), (2.5), (2.8) and (A3), it follows that

$$
\begin{aligned}
& -x_{n}^{\prime \prime}(t) \leq p(t) h_{1}\left(\theta\left(x_{n}(t)\right)\right) k_{1}\left(\theta\left(y_{n}(t)\right)\right)\left(u_{1}\left(x_{n}^{\prime}(t)\right)+v_{1}\left(x_{n}^{\prime}(t)\right)\right), \quad t \in(0,1), \\
& -y_{n}^{\prime \prime}(t) \leq q(t) h_{2}\left(\theta\left(x_{n}(t)\right)\right) k_{2}\left(\theta\left(y_{n}(t)\right)\right)\left(u_{2}\left(y_{n}^{\prime}(t)\right)+v_{2}\left(y_{n}^{\prime}(t)\right)\right), \quad t \in(0,1),
\end{aligned}
$$

which implies that

$$
\begin{aligned}
& \frac{-x_{n}^{\prime \prime}(t)}{u_{1}\left(x_{n}^{\prime}(t)\right)+v_{1}\left(x_{n}^{\prime}(t)\right)} \leq h_{1}(M) k_{1}(M) p(t), \quad t \in(0,1), \\
& \frac{-y_{n}^{\prime \prime}(t)}{u_{2}\left(y_{n}^{\prime}(t)\right)+v_{2}\left(y_{n}^{\prime}(t)\right)} \leq h_{2}(M) k_{2}(M) q(t), \quad t \in(0,1),
\end{aligned}
$$

Integrating from $t$ to 1 , using the BCs, we obtain

$$
\begin{gathered}
\int_{t}^{x_{n}^{\prime}(t)} \frac{d z}{u_{1}(z)+v_{1}(z)} \leq h_{1}(M) k_{1}(M) \int_{t}^{1} p(s) d s, \quad t \in[0,1], \\
\int_{t}^{y_{n}^{\prime}(t)} \frac{d z}{u_{2}(z)+v_{2}(z)} \leq h_{2}(M) k_{2}(M) \int_{t}^{1} q(s) d s, \quad t \in[0,1],
\end{gathered}
$$


which can also be written as

$$
\begin{aligned}
& I\left(x_{n}^{\prime}(t)\right)-I(1 / n) \leq h_{1}(M) k_{1}(M) \int_{0}^{1} p(s) d s, \quad t \in[0,1], \\
& J\left(y_{n}^{\prime}(t)\right)-J(1 / n) \leq h_{2}(M) k_{2}(M) \int_{0}^{1} q(s) d s, \quad t \in[0,1] .
\end{aligned}
$$

The increasing property of $I$ and $J$ leads to

$$
\begin{aligned}
& x_{n}^{\prime}(t) \leq I^{-1}\left(h_{1}(M) k_{1}(M) \int_{0}^{1} p(s) d s+I(\varepsilon)\right), \quad t \in[0,1], \\
& y_{n}^{\prime}(t) \leq J^{-1}\left(h_{2}(M) k_{2}(M) \int_{0}^{1} q(s) d s+J(\varepsilon)\right), \quad t \in[0,1] .
\end{aligned}
$$

Integrating from 0 to $t$, using the BCs and (2.10), we obtain

$$
\begin{aligned}
& x_{n}^{\prime}(t) \leq I^{-1}\left(h_{1}(M) k_{1}(M) \int_{0}^{1} p(s) d s+I(\varepsilon)\right) \\
& +\frac{b_{1}}{a_{1}} J^{-1}\left(h_{2}(M) k_{2}(M) \int_{0}^{1} q(s) d s+J(\varepsilon)\right), \quad t \in[0,1], \\
& y_{n}^{\prime}(t) \leq \frac{b_{2}}{a_{2}} I^{-1}\left(h_{1}(M) k_{1}(M) \int_{0}^{1} p(s) d s+I(\varepsilon)\right) \\
& +J^{-1}\left(h_{2}(M) k_{2}(M) \int_{0}^{1} q(s) d s+J(\varepsilon)\right), \quad t \in[0,1] .
\end{aligned}
$$

From (2.11) and (2.7), it follows that

$$
\begin{aligned}
& M \leq\left\|x_{n}\right\|+\left\|y_{n}\right\| \leq\left(1+\frac{b_{2}}{a_{2}}\right) I^{-1}\left(h_{1}(M) k_{1}(M) \int_{0}^{1} p(s) d s+I(\varepsilon)\right) \\
& +\left(1+\frac{b_{1}}{a_{1}}\right) J^{-1}\left(h_{2}(M) k_{2}(M) \int_{0}^{1} q(s) d s+J(\varepsilon)\right)=r,
\end{aligned}
$$

which implies that

$$
\frac{M}{r} \leq 1
$$

a contradiction to (2.2). Hence, $\left\|x_{n}\right\|+\left\|y_{n}\right\|<M$.

Thus, $\left(x_{n}, y_{n}\right)$ is a solution of the following coupled system of BVPs

$$
\begin{aligned}
& -x^{\prime \prime}(t)=p(t) f\left(t, x(t), y(t), x^{\prime}(t)\right), \quad t \in(0,1), \\
& -y^{\prime \prime}(t)=q(t) g\left(t, x(t), y(t), y^{\prime}(t)\right), \quad t \in(0,1), \\
& a_{1} x(0)-b_{1} y^{\prime}(0)=0, x^{\prime}(1)=\frac{1}{n}, \\
& a_{2} y(0)-b_{2} x^{\prime}(0)=0, y^{\prime}(1)=\frac{1}{n},
\end{aligned}
$$

satisfying

$$
\begin{aligned}
& \left(t+\frac{b_{1}}{a_{1}}\right) \frac{1}{n} \leq x_{n}(t)<M, \frac{1}{n} \leq x_{n}^{\prime}(t)<L, \quad t \in[0,1], \\
& \left(t+\frac{b_{2}}{a_{2}}\right) \frac{1}{n} \leq y_{n}(t)<M, \frac{1}{n} \leq y_{n}^{\prime}(t)<L, \quad t \in[0,1] .
\end{aligned}
$$

We claim that

$$
\begin{aligned}
& x_{n}^{\prime}(t) \geq C_{1}^{\gamma_{1}} C_{2}^{\delta_{1}} \int_{t}^{1} p(s) \varphi_{M L}(s) d s, \\
& y_{n}^{\prime}(t) \geq C_{1}^{\gamma_{2}} C_{2}^{\delta_{2}} \int_{t}^{1} q(s) \psi_{M L}(s) d s,
\end{aligned}
$$

where

$$
\begin{aligned}
C_{1}= & \left(\frac{b_{2}}{a_{2}} \int_{0}^{1} p(s) \varphi_{M L}(s) d s\right)^{\frac{\delta_{1}}{\left(1-\gamma_{1}\right)\left(1-\gamma_{2}\right)-\delta_{1} \delta_{2}}} \\
& \left(\frac{b_{1}}{a_{1}} \int_{0}^{1} q(s) \psi_{M L}(s) d s\right)^{\frac{1-\gamma_{2}}{\left(1-\gamma_{1}\right)\left(1-\gamma_{2}\right)-\delta_{1} \delta_{2}}}, \\
C_{2}= & \left(\frac{b_{2}}{a_{2}} \int_{0}^{1} p(s) \varphi_{M L}(s) d s\right)^{\frac{1-\gamma_{1}}{\left(1-\gamma_{1}\right)\left(1-\gamma_{2}\right)-\delta_{1} \delta_{2}}} \\
& \left(\frac{b_{1}}{a_{1}} \int_{0}^{1} q(s) \psi_{M L}(s) d s\right)^{\frac{\delta_{2}}{\left(1-\gamma_{1}\right)\left(1-\gamma_{2}\right)-\delta_{1} \delta_{2}}} .
\end{aligned}
$$

To prove (2.14), consider the following relation

$$
\begin{aligned}
x_{n}(t) & =\left(t+\frac{b_{1}}{a_{1}}\right) \frac{1}{n}+\int_{0}^{\mathrm{t}} s p(s) f\left(s, x_{n}(s), y_{n}(s), x_{n}^{\prime}(s)\right) d s \\
& +\int_{\mathrm{t}}^{1} t p(s) f\left(s, x_{n}(s), y_{n}(s), x_{n}^{\prime}(s)\right) d s \\
& +\frac{b_{1}}{a_{1}} \int_{\mathrm{t}}^{1} q(s) g\left(s, x_{n}(s), y_{n}(s), y_{n}^{\prime}(s)\right) d s,
\end{aligned}
$$

which implies that

$$
x_{n}(0)=\frac{b_{1}}{a_{1}} \frac{1}{n}+\frac{b_{1}}{a_{1}} \int_{0}^{1} q(s) g\left(s, x_{n}(s), y_{n}(s), y_{n}^{\prime}(s)\right) d s .
$$

Using (A6) and (2.13), we obtain

$$
\begin{aligned}
& x_{n}(0) \geq \frac{b_{1}}{a_{1}} \int_{0}^{1} q(s) \psi_{M L}(s)\left(x_{n}(s)\right)^{\gamma_{1}}\left(y_{n}(s)\right)^{\delta_{1}} d s \\
& \geq\left(x_{n}(0)\right)^{\gamma_{1}}\left(y_{n}(0)\right)^{\delta_{1}} \frac{b_{1}}{a_{1}} \int_{0}^{1} q(s) \psi_{M L}(s) d s,
\end{aligned}
$$

which implies that

$$
x_{n}(0) \geq\left(y_{n}(0)\right)^{\frac{\delta_{1}}{1-\gamma_{1}}}\left(\frac{b_{1}}{a_{1}} \int_{0}^{1} q(s) \psi_{M L}(s) d s\right)^{\frac{1}{1-\gamma_{1}}} .
$$


Similarly, using (A6) and (2.13), we obtain

$$
y_{n}(0) \geq\left(x_{n}(0)\right)^{\frac{\delta_{2}}{1-\gamma_{2}}}\left(\frac{b_{2}}{a_{2}} \int_{0}^{1} p(s) \varphi_{M L}(s) d s\right)^{\frac{1}{1-\gamma_{2}}} .
$$

Now, using (2.18) in (2.17), we have

$$
\begin{aligned}
x_{n}(0) \geq & \left(x_{n}(0)\right)^{\frac{\delta_{1} \delta_{2}}{\left(1-\gamma_{1}\right)\left(1-\gamma_{2}\right)}}\left(\frac{b_{2}}{a_{2}} \int_{0}^{1} p(s) \varphi_{M L}(s) d s\right)^{\frac{\delta_{1}}{\left(1-\gamma_{1}\right)\left(1-\gamma_{2}\right)}} \\
& \left(\frac{b_{1}}{a_{1}} \int_{0}^{1} q(s) \psi_{M L}(s) d s\right)^{\frac{1}{1-\gamma_{1}}} .
\end{aligned}
$$

Hence,

$$
x_{n}(0) \geq C_{1} \text {. }
$$

Similarly, using (2.17) in (2.18), we obtain

$$
y_{n}(0) \geq C_{2} \text {. }
$$

Now, from (2.16), it follows that

$$
x_{n}^{\prime}(t) \geq \int_{\mathrm{t}}^{1} p(s) f\left(s, x_{n}(s), y_{n}(s), x_{n}^{\prime}(s)\right) d s .
$$

and using (A6), (2.13), (2.19) and (2.20), we obtain (2.14).

Similarly, we can prove (2.15).

Now, using (2.12), (A3), (2.13), (2.14) and (2.15), we have

$$
\begin{aligned}
& 0 \leq-x_{n}^{\prime \prime}(t) \leq h_{1}(M) k_{1}(M) p(t) \\
& \left(u_{1}\left(C_{1}^{\gamma_{1}} C_{2}^{\delta_{1}} \int_{\mathrm{t}}^{1} p(s) \varphi_{M L}(s) d s\right)+v_{1}(L)\right), \quad t \in(0,1), \\
& 0 \leq-y_{n}^{\prime \prime}(t) \leq h_{2}(M) k_{2}(M) q(t) \\
& \left(u_{2}\left(C_{1}^{\gamma_{2}} C_{2}^{\delta_{2}} \int_{\mathrm{t}}^{1} q(s) \psi_{M L}(s) d s\right)+v_{2}(L)\right), \quad t \in(0,1) .
\end{aligned}
$$

In view of (2.13), (2.21), (A1) and (A7), it follows that the sequences $\left\{\left(x_{n}^{(j)}, y_{n}^{(j)}\right)\right\}(j=0,1)$ are uniformly bounded and equicontinuous on $[0,1]$. Hence, by Arzela-Ascoli theorem, there exist subsequences $\quad\left\{\left(x_{n_{k}}^{(j)}, y_{n_{k}}^{(j)}\right)\right\}(j=0,1) \quad$ of $\left\{\left(x_{n}^{(j)}, y_{n}^{(j)}\right)\right\}(j=0,1)$ and $(x, y) \in C^{1}[0,1] \times C^{1}[0,1]$ such that $\left(x_{n_{k}}^{(j)}, y_{n_{k}}^{(j)}\right)$ converges uniformly to $\left(x^{(j)}, y^{(j)}\right)$ on $[0,1](j=0,1)$. Also, $a_{1} x(0)-b_{1} y^{\prime}(0)=a_{2} y(0)-b_{2} x^{\prime}(0)=x^{\prime}(1)=y^{\prime}(1)=0$.

Moreover, from (2.14) and (2.15), with $n_{k}$ in place of $n$ and taking $\lim n_{k} \rightarrow \infty$, we have

$$
\begin{aligned}
& x^{\prime}(t) \geq C_{1}^{\gamma_{1}} C_{2}^{\delta_{1}} \int_{t}^{1} p(s) \varphi_{M L}(s) d s, \\
& y^{\prime}(t) \geq C_{1}^{\gamma_{2}} C_{2}^{\delta_{2}} \int_{t}^{1} q(s) \psi_{M L}(s) d s,
\end{aligned}
$$

which shows that $x^{\prime}>0$ and $y^{\prime}>0$ on $[0,1), x>0$ and $y>0$ on $[0,1]$. Further, $\left(x_{n_{k}}, y_{n_{k}}\right)$ satisfy

$x_{n_{k}}^{\prime}(t)=x_{n_{k}}^{\prime}(0)-\int_{0}^{t} p(s) f\left(s, x_{n_{k}}(s), y_{n_{k}}(s), x_{n_{k}}^{\prime}(s)\right) d s, t \in[0,1]$,

$y_{n_{k}}^{\prime}(t)=y_{n_{k}}^{\prime}(0)-\int_{0}^{\mathrm{t}} q(s) g\left(s, x_{n_{k}}(s), y_{n_{k}}(s), y_{n_{k}}^{\prime}(s)\right) d s, t \in[0,1]$.

Passing to the limit as $n_{k} \rightarrow \infty$, we obtain

$$
\begin{aligned}
& x^{\prime}(t)=x^{\prime}(0)-\int_{0}^{t} p(s) f\left(s, x(s), y(s), x^{\prime}(s)\right) d s, \quad t \in[0,1], \\
& y^{\prime}(t)=y^{\prime}(0)-\int_{0}^{\mathrm{t}} q(s) g\left(s, x(s), y(s), y^{\prime}(s)\right) d s, \quad t \in[0,1],
\end{aligned}
$$

which implies that

$$
\begin{array}{ll}
-x^{\prime \prime}(t)=p(t) f\left(t, x(t), y(t), x^{\prime}(t)\right), & t \in(0,1), \\
-y^{\prime \prime}(t)=q(t) g\left(t, x(t), y(t), y^{\prime}(t)\right), & t \in(0,1) .
\end{array}
$$

Hence, $(x, y)$ is a $C^{1}$-positive solution of the system (1.5).

Example 2.2. Consider the following coupled system of singular BVPs

$$
\begin{aligned}
& -x^{\prime \prime}(t)=v^{\alpha_{1}+1}(x(t))^{\gamma_{1}}(y(t))^{\delta_{1}}\left(x^{\prime}(t)\right)^{-\alpha_{1}}, \quad t \in(0,1), \\
& -y^{\prime \prime}(t)=v^{\alpha_{2}+1}(x(t))^{\gamma_{2}}(y(t))^{\delta_{2}}\left(y^{\prime}(t)\right)^{-\alpha_{2}}, \quad t \in(0,1), \\
& x(0)-y^{\prime}(0)=y(0)-x^{\prime}(0)=x^{\prime}(1)=y^{\prime}(1)=0,
\end{aligned}
$$

where $0 \leq \gamma_{1}, \delta_{1}, \gamma_{2}, \delta_{2}<1$ satisfying $\left(1-\gamma_{1}\right)\left(1-\gamma_{2}\right) \neq \delta_{1} \delta_{2}$, $0<\alpha_{1}<1,0<\alpha_{2}<1$ and $v>0$ such that

$$
v<\sup _{c \in(0, \infty)} \frac{c}{2 \sum_{i=1}^{2}\left(\alpha_{i}+1\right)^{\frac{1}{\alpha_{i}+1}} c^{\frac{\gamma_{i}+\delta_{i}}{\alpha_{i}+1}}} .
$$

Choose $p(t)=q(t)=1, h_{1}(x)=v^{\alpha_{1}+1} x^{\gamma_{1}}, h_{2}(x)=v^{\alpha_{2}+1} x^{\gamma_{2}}$, $k_{1}(x)=x^{\delta_{1}}, k_{2}(x)=x^{\delta_{2}}, u_{1}(x)=x^{-\alpha_{1}}, u_{2}(x)=x^{-\alpha_{2}}$ and $v_{1}(x)=v_{2}(x)=0$. Then, $I(\mu)=\frac{\mu^{\alpha_{1}+1}}{\alpha_{1}+1}, J(\mu)=\frac{\mu^{\alpha_{2}+1}}{\alpha_{2}+1}$, $I^{-1}(\mu)=\left(\alpha_{1}+1\right)^{\frac{1}{\alpha_{1}+1}} \mu^{\frac{1}{\alpha_{1}+1}}$ and $J^{-1}(\mu)=\left(\alpha_{2}+1\right)^{\frac{1}{\alpha_{2}+1}} \mu^{\frac{1}{\alpha_{2}+1}}$.

Also, choose $\varphi_{M L}(t)=L^{-\alpha_{1}}, \psi_{M L}(t)=L^{-\alpha_{2}}$. Then,

$$
\begin{gathered}
\sup _{c \in(0, \infty)} \frac{c}{\left(1+\frac{b_{2}}{a_{2}}\right) I^{-1}\left(h_{1}(c) k_{1}(c) \int_{0}^{1} p(t) d t\right)}= \\
+\left(1+\frac{b_{1}}{a_{1}}\right) J^{-1}\left(h_{2}(c) k_{2}(c) \int_{0}^{1} q(t) d t\right) \\
\sup _{c \in(0, \infty)} \frac{c}{2 v \sum_{i=1}^{2}\left(\alpha_{i}+1\right)^{\frac{1}{\alpha_{i}+1}} c^{\frac{\gamma_{i}+\delta_{i}}{\alpha_{i}+1}}}>1 .
\end{gathered}
$$


Clearly, (A1) - (A7) are satisfied. Hence, by Theorem 2.1, the system (2.22) has at least one $C^{1}$-positive solution.

\section{References}

[1] R. P. Agarwal and D. O'Regan, Singular Differential and Integral Equations with Applications, Kluwer Academic Publishers, Dordrecht, 2003.

[2] S. Agmon, A. Douglis, L. Nirenberg, Estimates near the boundary for solutions of elliptic partial differential equations satisfying general boundary conditions II, Comm. Pure Appl. Math. 17 (1964) 35-92.

[3] H. Amann, Parabolic evolution equations with nonlinear boundary conditions, in: Nonlinear Functional Analysis and Its Applications, Berkeley, 1983, in: Proc. Sympos. Pure Math., vol. 45, Amer. Math. Soc., Providence, RI, 1986, pp. $17-27$.

[4] H. Amann, Parabolic evolution equations and nonlinear boundary conditions, J. Differential Equations 72 (1988) 201269.

[5] D.G. Aronson, A comparison method for stability analysis of nonlinear parabolic problems, SIAM Rev 20 (1978), 245-264.

[6] N.A. Asif, P.W. Eloe and R.A. Khan, Positive solutions for a system of singular second order nonlocal boundary value problems, Jounnal of the Korean Mathematical Society, 47 (5) (2010) $985-1000$.

[7] N.A. Asif and R.A. Khan, Positive solutions for a class of coupled system of singular three point boundary value problems, Boundary Value Problems 2009 (2009), Article ID 273063, 18 pages.

[8] N.A. Asif, R.A. Khan and J. Henderson, Existence of positive solutions to a system of singular boundary value problems, Dynamic Systems and Applications, 19 (2010) 395 - 404.
[9] X. Cheng and C. Zhong, Existence of positive solutions for a second-order ordinary differential system, J. Math. Anal. Appl. 312 (2005) 14-23.

[10] R.A. Khan and J.R.L. Webb, Existence of at least three solutions of nonlinear three point boundary value problems with super-quadratic growth, J. Math. Anal. Appl. 328 (2007) 690-698.

[11] A. Leung, A semilinear reactiondiffusion preypredator system with nonlinear coupled boundary conditions: Equilibrium and stability, Indiana Univ. Math. J. 31 (1982) 223-241.

[12] Y. Liu and B. Yan, Multiple solutions of singular boundary value problems for differential systems, J. Math. Anal. Appl. 287 (2003) 540-556.

[13] H. L"u, H. Yu and Y. Liu, Positive solutions for singular boundary value problems of a coupled system of differential equations, J. Math. Anal. Appl. 302 (2005), 14-29.

[14] F. Ali Mehmeti, Nonlinear Waves in Networks, Math. Res., vol. 80, Akademie-Verlag, Berlin, 1994.

[15] F. Ali Mehmeti, S. Nicaise, Nonlinear interaction problems, Nonlinear Anal. 20 (1993) 27-61.

[16] S. Nicaise, Polygonal Interface Problems, Methoden und Verfahren der Mathematischen Physik, vol. 39, Peter Lang, Frankfurt Main, 1993.

[17] Z. Wei, Positive solution of singular Dirichlet boundary value problems for second order differential equation system, J. Math. Anal. Appl. 328 (2007) 1255-1267.

[18] X. Xian, Existence and multiplicity of positive solutions for multi-parameter three-point differential equations system, J. Math. Anal. Appl. 324 (2006) 472-490.

[19] A. Zettl, Sturm-Liouvillie Theory, Math. Surveys Monogr., vol. 121, Amer. Math. Soc., Providence, RI, 2005. 